\title{
Actinidia chinensis Planch. root extract inhibits the proliferation, migration and invasion of breast cancer cells via the AKT/GSK-3 $\beta$ signaling pathway
}

\author{
Chunchun Gan ${ }^{1}$, Zhan Jin ${ }^{1}$, Xiaopeng Wei ${ }^{2}$, Meina Jin ${ }^{2}$ \\ ${ }^{1}$ School of Medicine, Quzhou College of Technology, Quzhou, China \\ ${ }^{2}$ School of Pharmacy, Tianjin Medical University, Tianjin, China
}

\begin{abstract}
Introduction. Actinidia chinensis Planch. root extract (acRoots), known as a traditional Chinese medicine (TCM), has shown antitumor efficacy in various types of human cancers. However, its role and underlying mechanisms in breast cancer $(\mathrm{BCa})$ have not been elucidated.

Material and methods. In the present study, the effects of acRoots on cell viability, apoptosis, migration and invasion were analyzed by MTT assay, colony formation, flow cytometry, wound healing and Transwell assay in MDA-MB-231 and MDA-MB-453 breast cancer cell lines. The expression levels of relevant proteins were determined by Western blot assay.

Results. The results revealed that acRoots inhibited proliferation, migration, and invasion and promoted apoptosis of BCa cells. Moreover, acRoots decreased the expression of cyclin D1, survivin, Bcl-2, N-cadherin, and Snail and increased the expression of Bax and E-cadherin in MDA-MB-231 and MDA-MB-453 cells. AcRoots inhibited the AKT/GSK- $3 \beta$ pathway by decreasing the levels of phosphorylated AKT, phosphorylated GSK- $3 \beta$ and $\beta$-catenin.

Conclusions. The described effects of acRoots on the cultured BCa cells suggest that they may be mediated by the inhibition of the AKT/GSK-3 $\beta$ signaling pathway. Thus, further studies are warranted to test the possibility that AcRoots may be used as a promising anticancer agent for breast cancer treatment. (Folia Histochemica et Cytobiologica 2021, Vol. 59, No. 4, 226-235)
\end{abstract}

Key words: Actinidia chinensis Planch. root; breast cancer cells; proliferation; apoptosis; migration; invasion; AKT/GSK-3 $\beta$

\section{Introduction}

Breast cancer $(\mathrm{BCa})$ is one of the most common malignancies, accounting for almost 450,000 deaths annually worldwide [1-4]. The current treatments for $\mathrm{BCa}$ are mainly surgical resection and chemotherapy. Despite improvements in clinical strategies, including early diagnosis, surgical resection, hormonal therapy, radiotherapy and chemotherapy, long-term clinical outcomes and mortality rates remain unsatisfactory

Correspondence address: Meina Jin,

22 Fengtai Road, Heping District, Tianjin, China, 300070

e-mail: songjinmeina@163.com
[5]. Among these factors, severe side effects and multidrug resistance caused by chemotherapy are the main obstacles that lead to poor therapeutic effects and survival rates [6]. The etiology, cell proliferation and metastasis of $\mathrm{BCa}$ are generally mediated by multiple mechanisms or pathways [7,8]. At present, several natural products extracted from a variety of sources are considered to be effective complementary therapeutic agents and have become a novel research focus for the treatment of cancer due to their regulation of a variety of physiological pathways [9-11].

Actinidia chinensis Planch. root extract (acRoots), a kind of traditional Chinese medicine (TCM), has various biological activities, including antioxidant, anti-in-

This article is available in open access under Creative Common Attribution-Non-Commercial-No Derivatives 4.0 International (CC BY-NC-ND 4.0) license, allowing to download articles and share them with others as long as they credit the authors and the publisher, but without permission to change them in any way or use them commercially. (C) Polish Society for Histochemistry and Cytochemistry 
flammatory, purification, immunological and anticancer activities [12-14]. Recently, acRoots was reported to inhibit in vitro the progression of hepatocellular carcinoma [15-18], gastric cancer [19], colon cancer [20] and lung cancer cell lines [21]. However, the effects of acRoots on $\mathrm{BCa}$ cells are still unknown, and the mechanism responsible for the anticancer effects is very limited.

In the present study, we first investigated the role of acRoots in $\mathrm{BCa}$ cell lines. In addition, mechanistic studies suggested that the inhibitory effect of acRoots on BCa may occur through blocking the AKT/GSK-3 $\beta$ signaling pathway, which would provide a potential strategy for developing new agents for the treatment of breast cancer.

\section{Materials and methods}

Preparation of acRoots. The roots of Actinidia chinensis Planch. were washed and chopped, suspended in 10 volumes of distilled water, and heated at $100^{\circ} \mathrm{C}$ for $1 \mathrm{~h}$. Then, the decoction was filtered. The entire extraction process was repeated twice. The filtrate was combined twice, and the concentrate was obtained in a ratio of 1:1, extracted three times with $n$-butanol. The two extracted filtrates were combined, the solvent was recovered to dryness, and the resulting extracts were mixed and equilibrated to $1 \mathrm{~g} / \mathrm{mL}$ mother solution. A stock solution of acRoots was prepared by dissolving the sample in dimethyl sulfoxide (DMSO, Gibco, Thermo Fisher Scientific, Waltham, MA, USA) and diluted in Dulbecco's modified Eagle's medium (DMEM, Gibco) to make the final DMSO concentration of $0.1 \%$. The active acRoots solution was continuously diluted at the following concentrations: $0.1,1$, and $10 \mathrm{mg} / \mathrm{mL}$ or $0.1,1$, and $10 \mu \mathrm{g} / \mathrm{mL}$. The wells with only DMEM were used as the negative control, whereas wells with DMEM and $0.1 \%$ DMSO were used as the control (Ctrl group, i.e. DMSO group).

Cell culture and treatment. The human breast epithelial cell line MCF-10A and human BCa cell lines MDA-MB-231 and MDA-MB-453 were purchased from American Type Culture Collection (ATCC; Manassas, VA, USA) and cultured in DMEM (Gibco) with $10 \%$ fetal bovine serum (FBS, Gibco) and 1\% penicillin/streptomycin $(100 \mathrm{U} / \mathrm{mL}$ penicillin and $100 \mathrm{mg} / \mathrm{mL}$ streptomycin (Gibco) at $37^{\circ} \mathrm{C}$ in the atmosphere of $5 \% \mathrm{CO}_{2}$.

Cell viability assay. Cells $\left(5 \times 10^{4}\right.$ cells/well $)$ were seeded in 96-well culture plates. After treatment with different concentrations of acRoots for $24 \mathrm{~h}, 5 \mathrm{mg} / \mathrm{mL}$ of 3-(4,5-dimethyl-2-thiazolyl)-2,5-diphenyl-2-H-tetrazolium bromide (MTT, $20 \mu \mathrm{L} /$ /well) was added to the cells and incubated for $4 \mathrm{~h}$ at $37^{\circ} \mathrm{C}$. Then, $150 \mu \mathrm{L} /$ well of DMSO (Gibco) was added to dissolve crystals. The absorbance at $570 \mathrm{~nm}$ (OD570) was detected in a microplate reader (Bio-Rad, Hercules, CA, USA).
Colony formation assay. Colony formation assay was used to evaluate the effect of acRoots on cell growth. In brief, cells were seeded at a density of $1 \times 10^{3}$ cells/well in 6-well plates and were cultured with indicated doses of acRoots. Then, living cells $\left(2 \times 10^{3}\right.$ cells/well $)$ were cultured in different 6-well plates for an additional two weeks until cell colonies were visible. The cells were washed twice in phosphate-buffered saline (PBS), fixed in paraformaldehyde (PFA, SigmaAldrich, St. Louis, MO, USA) for $20 \mathrm{~min}$ and stained in $0.2 \%$ crystal violet (Solarbio, Beijing, China). Stained cells were washed in PBS and photographed after drying with a microscopic camera.

Cell apoptosis assay. Cell apoptosis was evaluated using the Annexin V-FITC Apoptosis Detection Kit (Catalog no. 88-8005-72, Invitrogen, Grand Island, NY, USA) following the manufacturer's instructions. In brief, the treated cells were digested with $0.025 \%$ trypsin and washed with PBS. Then, the cells were collected and resuspended in binding buffer and stained with $5 \mu \mathrm{L}$ annexin $\mathrm{V}$-FITC and $5 \mu \mathrm{L}$ propidium iodide (PI) in the dark for $15 \mathrm{~min}$ at room temperature (RT). The fluorescence was detected using flow cytometry with a FACScan flow cytometer (BD Biosciences, Franklin Lakes, NJ, USA).

Wound-healing assay. A wound healing assay was performed to test cell migration. $5 \times 10^{4}$ cells were seeded into 6 -well plates and maintained overnight at $37^{\circ} \mathrm{C}$ in the atmosphere of incubators with $5 \% \mathrm{CO}_{2}$. When cells reached $90 \%$ confluence, they were treated with $10 \mu \mathrm{g} / \mathrm{mL}$ mitomycin $\mathrm{C}$ (SigmaAldrich) for $2 \mathrm{~h}$ [22-24]. (Mitomycin C is used as a proliferation inhibitor to eliminate the contribution of cell division on wound closure to exclude the effect of reduced scratch area due to cell growth). The monolayer cells were scratched with a $200 \mu \mathrm{L}$ pipette tip on the bottom of each plate after cells reached $100 \%$ confluence. Then, the cell debris was washed away with PBS, and the remaining cells were cultured in serum-free medium with acRoots at the indicated doses for $24 \mathrm{~h}$. At $0 \mathrm{~h}$ and $24 \mathrm{~h}$ after scratching, the gap area width was captured using a microscope camera (Olympus, Tokyo, Japan).

Transwell assay. Transwell assays were performed to measure the cell invasion capacity. Cells were treated with or without acRoots for $24 \mathrm{~h}$ and then harvested. The cells $\left(5 \times 10^{4}\right.$ cells/well $)$ treated under the different conditions described above were seeded in the top chamber (Millipore, Billerica, MA, USA), which was precoated with Matrigel (BD Biosciences). Serum-free culture medium was added to the top chambers, while the bottom chambers were filled with $500 \mu \mathrm{L}$ of culture medium containing $10 \%$ FBS. After incubation for $24 \mathrm{~h}$, cells in the bottom chamber were fixed with $4 \%$ paraformaldehyde, stained with $0.1 \%$ crystal violet and counted under a microscope. 
Western blot assay. Cells were lysed with RIPA lysis buffer (Beyotime, Beijing, China). After quantification with a Pierce ${ }^{\mathrm{TM}}$ BCA Protein Assay Kit (Thermo Fisher Scientific), total proteins were fractionated by sodium dodecyl sulfate polyacrylamide gel electrophoresis (SDS-PAGE). Then, proteins were transferred onto a PVDF membrane (Millipore, Bedford, MA, USA), blocked with 5\% skim milk, and incubated with the following primary antibodies at $4^{\circ} \mathrm{C}$ overnight: anti-cyclin D1 (1:1000, \#ab92552, Abcam), anti-survivin (1:1000, \#ab32072, Abcam), anti-Bcl-2 (1:800, \#ab59348, Abcam), anti-Bax (1:2000, \#ab32503, Abcam), anti-E-cadherin (1:500, \#ab15148, Abcam), anti-N-cadherin (1:1000, \#ab76057, Abcam), anti-Snail (1:500, \#ab180714, Abcam), anti-p-GSK-3 $\beta$ (1:10000, \#ab75814, Abcam), antiGSK-3 $\beta$ (1:5000, \#ab32391, Abcam), anti-p-AKT (1:1000, \#ab38449, Abcam), anti-AKT (1:500, \#ab8805, Abcam) and anti-GAPDH (1:2000, \#ab181602, Abcam). Subsequently, the membranes were incubated with HRP-conjugated goat anti-rabbit IgG antibody or anti-mouse IgG antibody (1:2,000; Abcam, Cambridge, MA, USA) for $1 \mathrm{~h}$ at RT. The expression of proteins was detected and analyzed with an imaging system (Bio-Rad) and ImageJ software (NIH Image, Bethesda, MD, USA).

Statistical analysis. Statistical product and service solutions (SPSS, version 19.0, IBM, New York, NY, USA) and GraphPad Prism 7 (Graphpad Inc., San Diego, CA, USA) were used to conduct data analysis. All results are displayed as the mean \pm SD of from three independent experiments, and mean values were compared using Student's $t$-test or one-way ANOVA with Tukey's post hoc test, respectively. All experiments were repeated more than three times, and a $p$ value $<0.05$ was considered statistically significant.

\section{Results}

\section{AcRoots inhibits the proliferation of $\mathrm{BCa}$ cells}

To investigate the anticancer effects of acRoots, MTT assay was performed to evaluate the proliferation of the breast cancer cell lines MDA-MB-231 and MDA-MB-453 and the normal breast epithelial cell line MCF10A. Analysis revealed that acRoots $(0.1,1,10 \mathrm{mg} / \mathrm{mL})$ treatment decreased the viability of both MDA-MB-231 and MDA-MB-453 cells in a dose-dependent manner (Fig. 1A) but did not have any effect on the viability of MCF-10A cells (Fig. 1A). The inhibitory effect of acRoots on the proliferation of BCa cells was also confirmed by a colony formation assay (Fig. 1B). Similarly, cell colony numbers in the acRoots treatment groups were lower than those in the control and DMSO groups, and that in the $10 \mathrm{mg} / \mathrm{mL}$ acRoots group had the most significant reduction (Fig. 1B). These data indicated that acRoots inhibits the proliferation of $\mathrm{BCa}$ cells in vitro.

\section{AcRoots promotes apoptosis of BCa cells}

We further explored the effect of acRoots on the apoptosis of BCa cells by flow cytometry. The apoptotic cell percentages in the control, DMSO, acRoots $(0.1 \mathrm{mg} / \mathrm{mL})$, acRoots $(1 \mathrm{mg} / \mathrm{mL})$ and acRoots $(10 \mathrm{mg} / \mathrm{mL})$ groups were $7.67 \pm 1.13 \%, 8.12 \pm 1.00 \%$, $12.79 \pm 1.89 \%, 15.72 \pm 2.07 \%, 22.89 \pm 3.37 \%$ in MDA-MB-231 cells and $5.60 \pm 0.72 \%, 6.97 \pm 1.80 \%$, $13.42 \pm 2.30 \%, 19.56 \pm 2.05 \%, 24.86 \pm 4.64 \%$ in MDA-MB-453 cells, respectively (Fig. 2A, B). The percentages of apoptotic cells in the acRoots groups were significantly increased compared with those in the Ctrl and DMSO groups (Fig. 2A, B) in both MDA-MB-231 and MDA-MB-453 cells, indicating that acRoots promotes apoptosis of $\mathrm{BCa}$ cells.

\section{AcRoots inhibits the migration and invasion of BCa cells}

The effect of acRoots on the migration ability of BCa cells was measured by wound healing assay. By detecting the changes in the spacing between cells in each group at 0 and $24 \mathrm{~h}$ after scratching, we found that acRoots suppressed the migratory capacity of both MDA-MB-231 and MDA-MB-453 cells (Fig. 3A). Transwell invasion assays revealed that acRoots significantly reduced the number of invasive cells (Fig. 3B). Thus, acRoots decreased the migration and invasion of $\mathrm{BCa}$ cells.

\section{AcRoots regulates the expression of proliferation-, apoptosis- and epithelial-mesenchymal transition (EMT)-related factors in breast cancer cells}

Since the above results showed that acRoots inhibited proliferation, migration and invasion and induced apoptosis of BCa cells, the levels of proteins related to cell proliferation (survivin and cyclin D1), apoptosis (Bcl-2 and Bax), and EMT (N-cadherin, E-cadherin and Snail) were determined by western blot assay. Consistently, following treatment with acRoots $(0.1,1$ and $10 \mathrm{mg} / \mathrm{mL})$, the protein levels of survivin, cyclin D1, N-cadherin, Bcl-2 and Snail were significantly decreased; however, the protein levels of Bax and E-cadherin were significantly increased in a dose-dependent manner in MDA-MB-231 (Fig. 4A) and MDA-MB-453 (Fig. 4B) cells.

\section{AcRoots inhibits the AKT/GSK-3 $\beta$ signaling pathway in breast cancer cells}

Furthermore, we examined AKT/GSK- $3 \beta$ signaling, which plays a critical role in the regulation of cell proliferation, apoptosis and EMT. Western blot assay revealed that acRoots treatment significantly reduced the levels of phosphorylated AKT, phosphorylated GSK- $3 \beta$ and $\beta$-catenin, whereas it exhibited no effect 


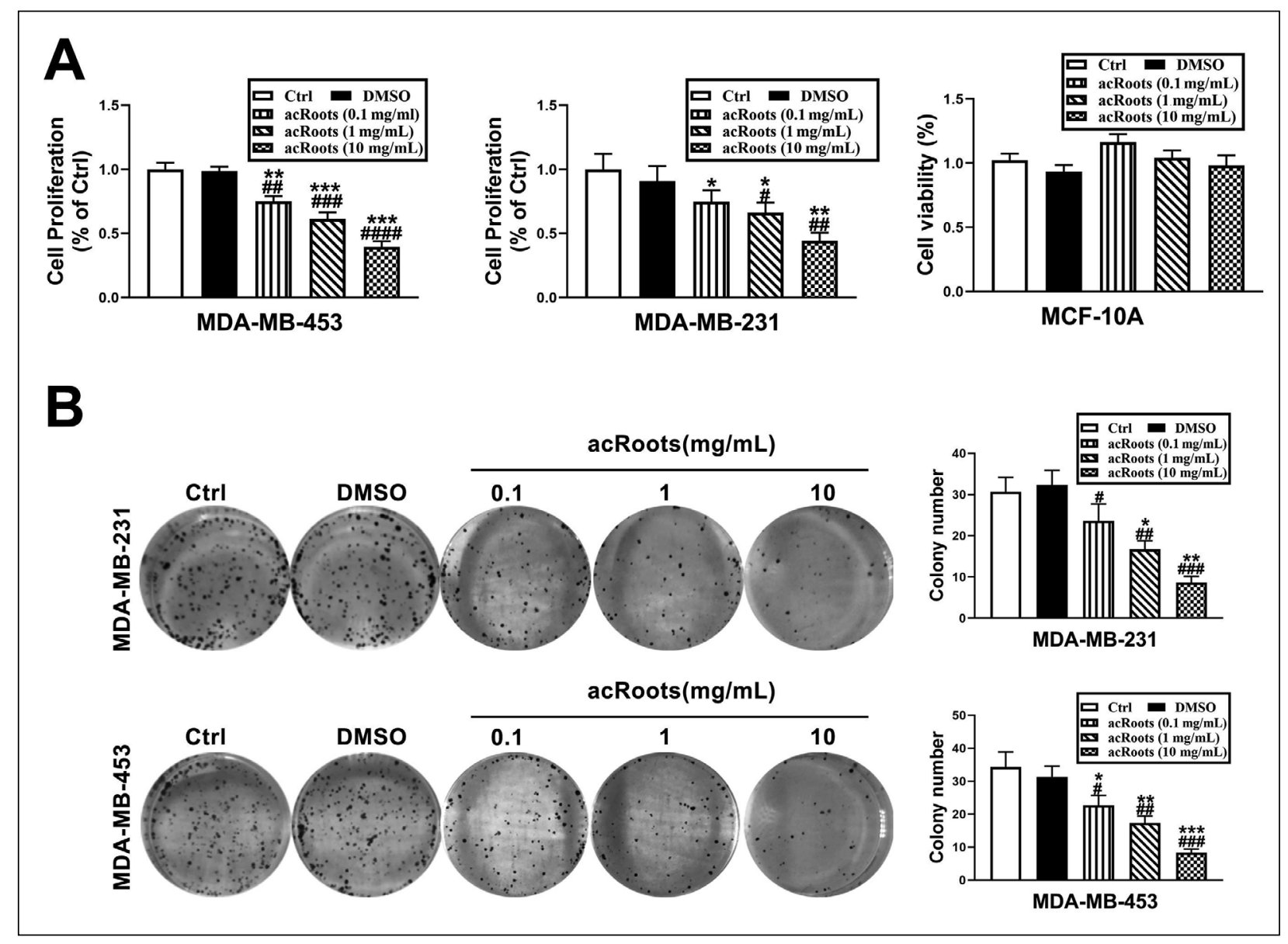

Figure 1. AcRoots inhibits the proliferation of BCa cells. A-B. After treatment with different concentrations of AcRoots for $24 \mathrm{~h}$, cell proliferation (A) and colony formation (B) were assessed by MTT assay and colony formation assay. Values are displayed as the mean \pm SD of triplicate measurements from three independent experiments. ${ }^{*} \mathrm{p}<0.05$, $*$ * $<<0.01$, ***p $<0.001$, compared with the blank control (Ctrl) group; ${ }^{\#} \mathrm{p}<0.05,{ }^{\# \#} \mathrm{p}<0.01$, ${ }^{\# \#} \mathrm{p}<0.001$, compared with the DMSO group.

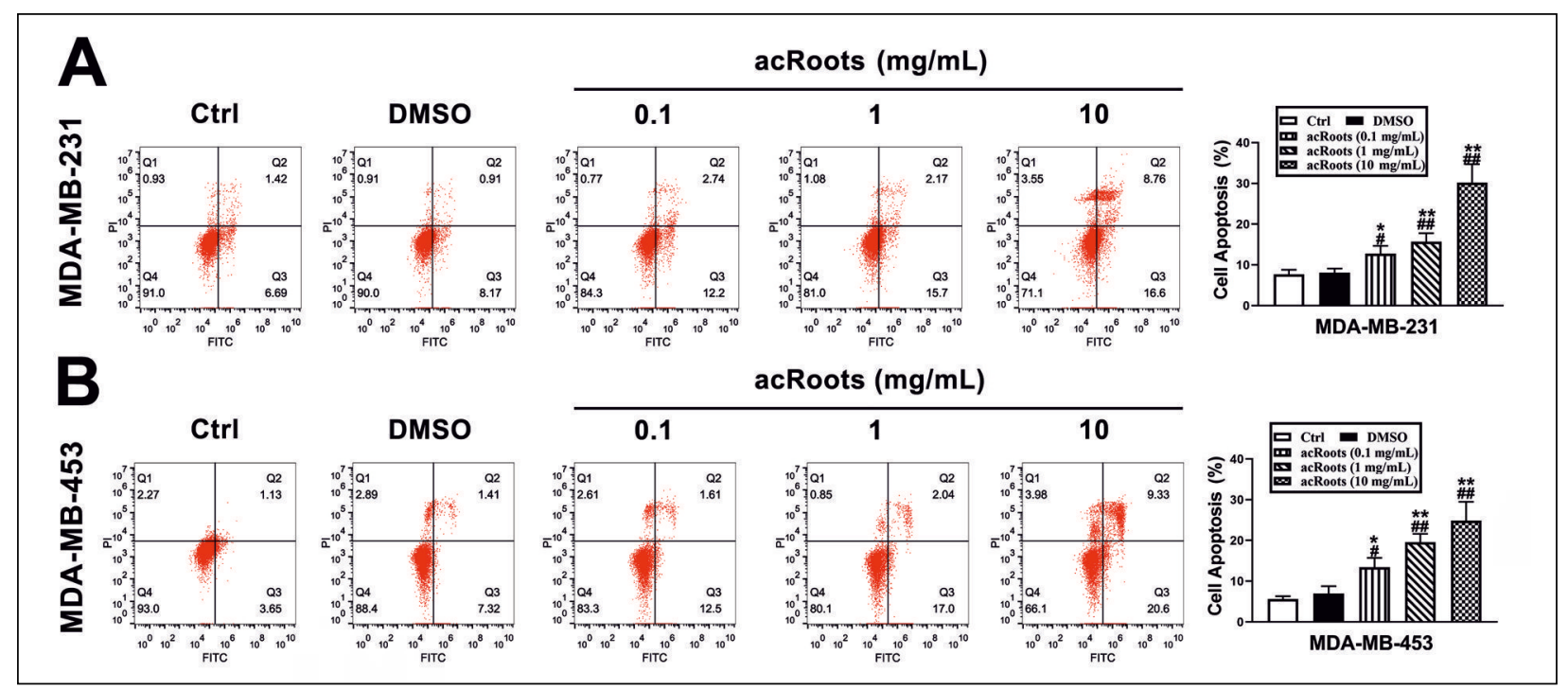

Figure 2. AcRoots promotes the apoptosis of BCa cells. A-B. Cell apoptosis induced by acRoots in MDA-MB-231 (A) and MDA-MB-453 (B) cell lines was analyzed by flow cytometry with FITC-annexin V/PI staining. These data are representative of triplicate independent experiments conducted under the same conditions. ${ }^{*} \mathrm{p}<0.05,{ }^{* *} \mathrm{p}<0.01$, ${ }^{* * *} \mathrm{p}<0.001$, compared with the blank control (Ctrl) group; ${ }^{\#} \mathrm{p}<0.05,{ }^{\# \#} \mathrm{p}<0.01,{ }^{\# \#} \mathrm{p}<0.001$, compared with the DMSO group. 


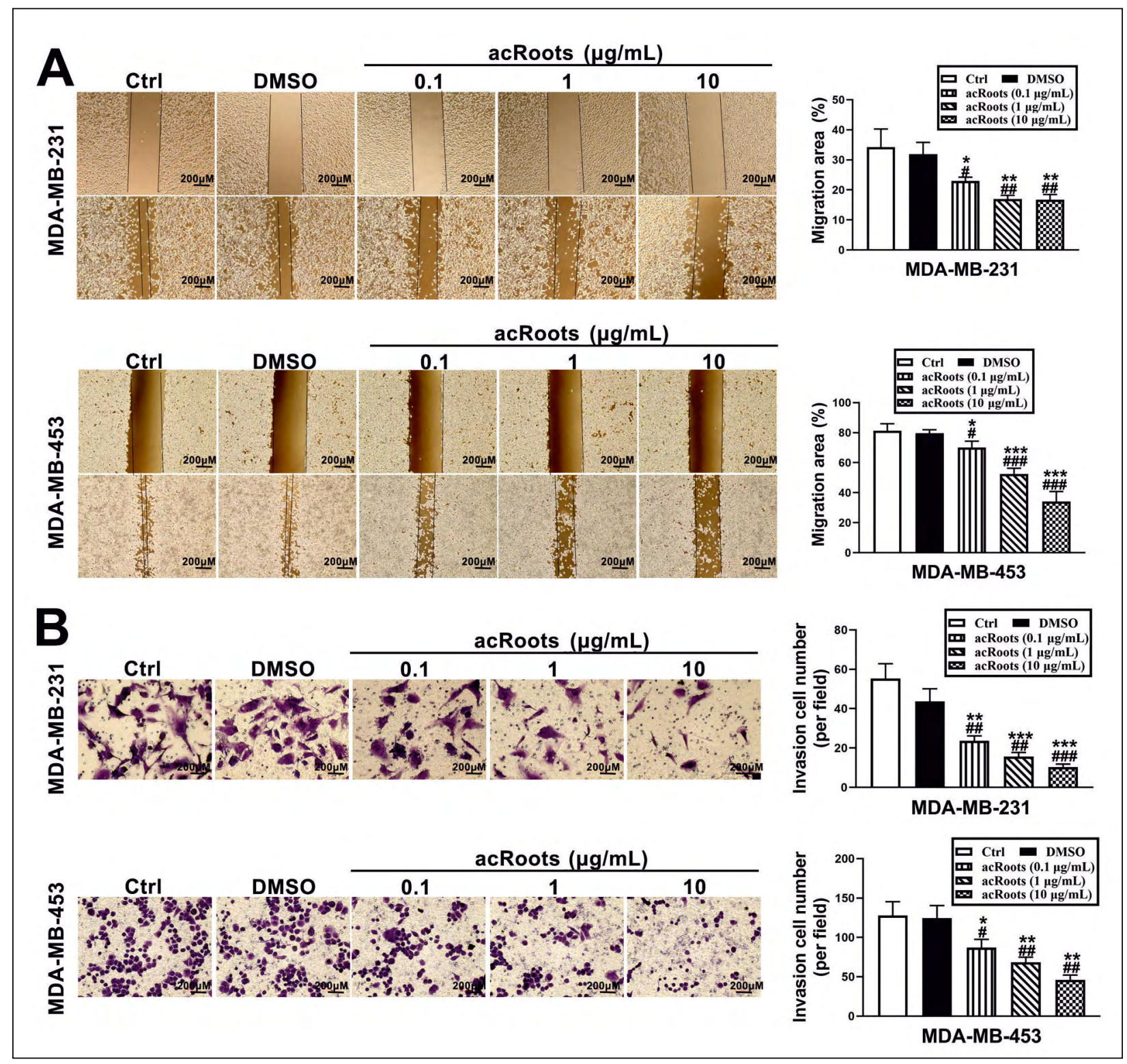

Figure 3. AcRoots inhibits the migration and invasion of BCa cells. A-B. Cell migration (A) and (B) invasion were detected using wound healing and Transwell assays (magnification, $\times 10$; scale bar, $200 \mu \mathrm{m}$ ), respectively, when MDA-MB-231 and MDA-MB-453 cells were treated with different concentrations of acRoots. These data are representative of triplicate independent experiments conducted under the same conditions. ${ }^{*} \mathrm{p}<0.05,{ }^{* *} \mathrm{p}<0.01,{ }^{* * *} \mathrm{p}<0.001$, compared with the blank control (Ctrl) group; ${ }^{\#} \mathrm{p}<0.05,{ }^{\# \#} \mathrm{p}<0.01,{ }^{\# \# \#} \mathrm{p}<0.001$, compared with the DMSO group.

on the total levels of AKT and GSK-3 $\beta$ in any of the treatment groups in MDA-MB-231 (Fig. 5A) and MDA-MB-453 (Fig. 5B) cells.

\section{Discussion}

Breast cancer is one of the most aggressive cancers, and a high metastasis rate is the main cause of death [30]. Therefore, exploring the mechanism of the proliferation and metastasis of $\mathrm{BCa}$ cells is beneficial for targeted therapy of $\mathrm{BCa}$. Accumulated evidence shows that, as a complementary therapy for $\mathrm{BCa}$, TCM has certain advantages in inhibiting the proliferation and metastasis of cancer cells [31, 32]. In this study, we demonstrated that acRoots effectively inhibited cell viability, migration, and invasion and induced apoptosis in BCa cells. Moreover, acRoots regulated the expression of proliferation-, EMT- and apoptosis-related markers.

Inhibiting the proliferation and promoting the apoptosis of cancer cells is a promising strategy for tumor therapy [33]. Overall, drug-induced apoptosis 


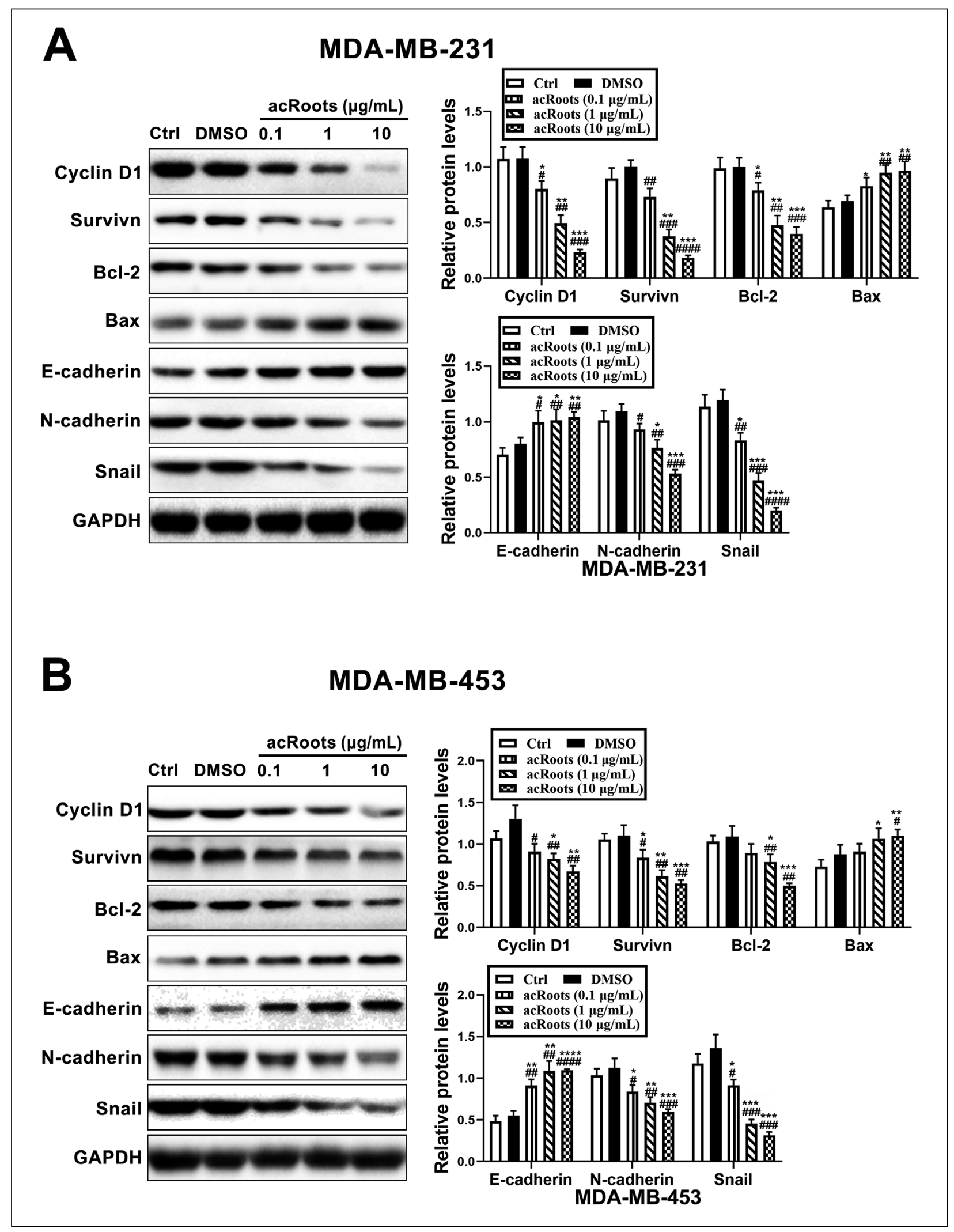

Figure 4. AcRoots regulates the expression of proliferation-, apoptosis- and EMT-related factors in BCa cells. A-B. The protein levels of cell proliferation-, apoptosis- and EMT-related factors were evaluated using Western blot assay when MDA-MB-231 (A) and MDA-MB-453 (B) cells were treated with different concentrations of acRoots. GAPDH was used as a protein loading control. These data are representative of triplicate independent experiments conducted under the same conditions. ${ }^{*} \mathrm{p}<0.05,{ }^{*} \mathrm{p}<0.01,{ }^{* * *} \mathrm{p}<0.001$, compared with the blank control (Ctrl) group; ${ }^{*} \mathrm{p}<0.05,{ }^{\# \#} \mathrm{p}<0.01$, $\# \#$ p $<0.001$, compared with the DMSO group. 


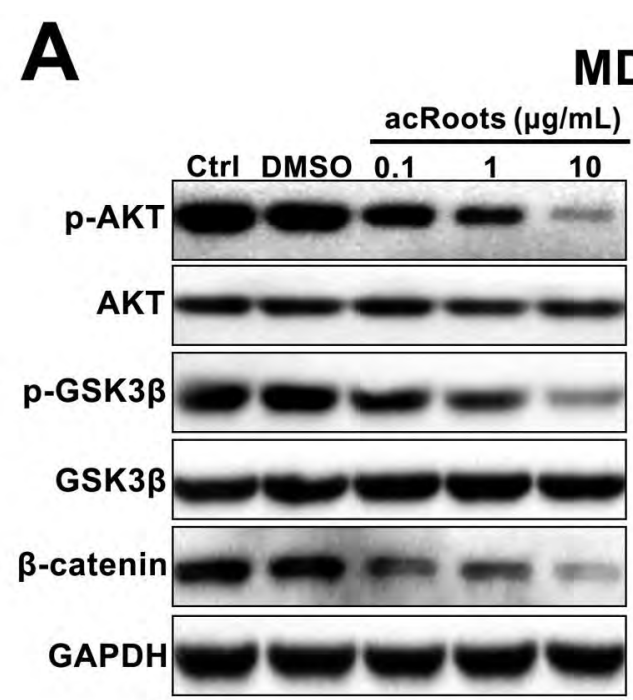

\section{MDA-MB-231}

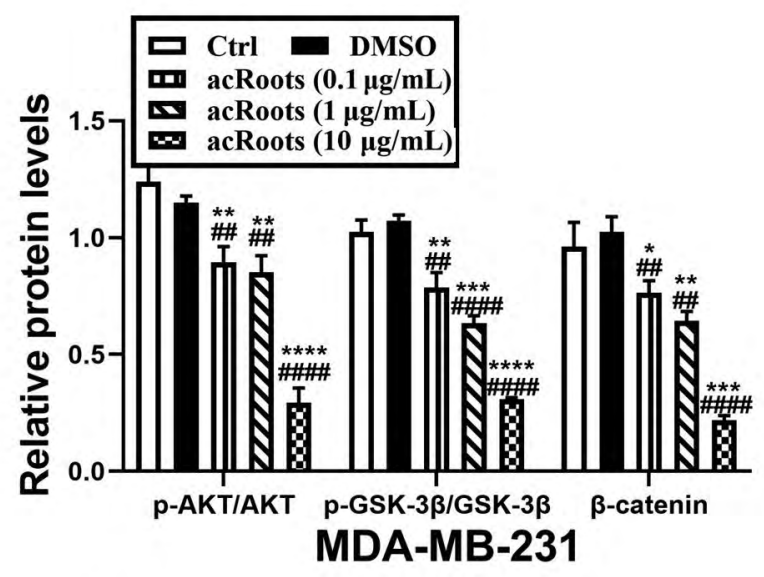

B

MDA-MB-453
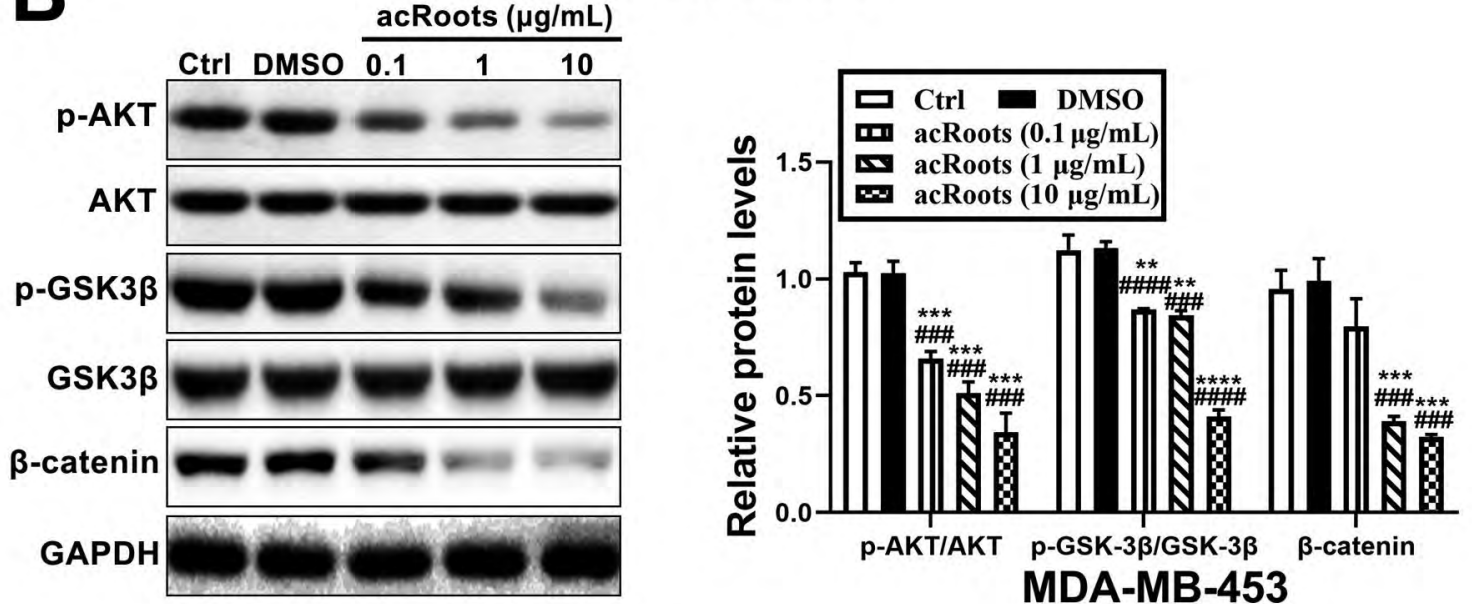

Figure 5. AcRoots inhibits the AKT/GSK- $3 \beta$ signaling pathway in BCa cells. A-B. The protein levels of p-Akt, Akt, GSK-3 $\beta$, and p-GSK-3 $\beta$ in MDA-MB-231 and MDA-MB-453 cells were determined by Western blot analysis. GAPDH was used as a protein loa ding control. These data are representative of triplicate independent experiments conducted under the same conditions. ${ }^{*} \mathrm{p}<0.05,{ }^{* *} \mathrm{p}<0.01,{ }^{* * *} \mathrm{p}<0.001$, compared with the blank control (Ctrl) group; ${ }^{\#} \mathrm{p}<0.05$, ${ }^{\# \#} \mathrm{p}<0.01$, \#\#\# $\mathrm{p}<0.001$, compared with the DMSO group.

is one of the main mechanisms of action in the treatment of cancer. Hou et al. suggested that acRoots could inhibit proliferation and induce cell cycle arrest in S-phase and induce apoptosis in hepatocellular carcinoma cells [15]. Consistently, we also found that acRoots treatment significantly decreased cell viability but increased apoptosis rates, along with decreased Bcl-2 protein levels and increased Bax protein levels in BCa cells. A recent study found that the level of cleaved caspase 3 and cell apoptosis rate were significantly upregulated in the acRoots-treated group, whereas this effect was reversed in the co-treated group with acRoots + Z-VAD-FMK (a caspase inhibitor), suggesting that acRoots may inhibit gastric cancer cell proliferation in part by promoting apoptosis [34]. However, further studies are needed to determine whether acRoots inhibits the cell proliferation in $\mathrm{BCa}$ is caused by caspase-dependent apoptosis pathway. Growing evidence suggests that the mechanism associated with $\mathrm{BCa}$ invasion and metastasis involves the induction of EMT, which is characterized by the disappearance of epithelial phenotypes and the acquisition of fibroblast-like characteristics and is accompanied by a decline in the expression of adhesion molecules that maintain epithelial cell contacts [35,36]. The inhibition of EMT 
transcription factors (such as Snail) on genes encoding epithelial cell-specific proteins (such as E-cadherin) can promote a decrease in cell adhesion [37]. Previous studies have revealed that acRoots inhibits invasion and migration and EMT in hepatocellular carcinoma cells [17], and this effects of acRoots was confirmed in this study performed on breast cancer cell lines. In the present study, acRoots was also proven to inhibit the migration and invasion of BCa cells. Consistently, the decrease in protein levels of $\mathrm{N}$-cadherin and Snail and the increase in protein levels of E-cadherin also confirmed the above results. Taken together, acRoots may inhibit BCa progression by regulating cell proliferation, migration, invasion and EMT.

A previous study showed that acRoots inhibited lung cancer cell proliferation and apoptosis via the PI3K signaling pathway by decreasing the gene expression of PI3K family members [40]. Another recent study revealed that the polysaccharide components of Actinidia chinensis Plant played a protective effect in hypoxia-induced apoptosis of cardiomyocytes treated with angiotensin II by inhibiting the activation of the PI3K/AKT pathway [41]. Accordingly, we hypothesize that acRoots may regulate the progression of $\mathrm{BCa}$ cells by regulating AKT/GSK- $3 \beta$ signaling. Aberrant activation of the AKT/GSK- $3 \beta$ signaling pathway has been shown to be involved in the progression of many cancers $[26,28]$. With increasing evidence that the AKT/ /GSK- $3 \beta$ signaling pathway plays a key role in regulating $\mathrm{BCa}$ growth, survival, motility and drug resistance, there are now numerous data suggesting that various components of this pathway may be molecular targets for BCa treatment $[17,28]$. Thus, the AKT/GSK-3 $\beta$ signaling pathway is considered to be an attractive target for the development of new anticancer drugs for the treatment of BCa patients. As a downstream target of the PI3K/AKT pathway, GSK-3 $\beta$ may be inactivated by AKT-mediated phosphorylation of its Ser9 residue $[42,43]$. Phosphorylation of $\beta$-catenin at Ser33, Ser37 and Thr 41 residues by GSK-3 $\beta$ eventually leads to $\beta$-catenin sequestration and degradation, thereby inhibiting the $\mathrm{Wnt} / \beta$-catenin signaling pathway [44]. Thus, AKT activation leads to inhibition of GSK- $3 \beta$ kinase activity, which activates the Wnt $/ \beta$-catenin signaling pathway. In addition, $\mathrm{Wnt} / \beta$-catenin is one of the critical signaling pathways that regulates proliferation and EMT, and its downstream targets are survivin and cyclin D1 [45, 46]. Further western blot assays verified that acRoots treatment significantly decreased the levels of phosphorylated AKT, phosphorylated GSK-3 $\beta$ and $\beta$-catenin, and acRoots treatment also reduced the levels of survivin and cyclin D1 downstream target genes of $\mathrm{Wnt} / \beta$-catenin in MDA-MB-231 and MDA-MB-453 cells, indicating that acRoots suppresses the AKT/GSK- $3 \beta$ signaling pathway, which presumably contributes to the inhibition of proliferation, invasion and EMT of BCa cells.

At the same time, recent pharmacological studies have shown that acRoots has anti-tumor function in vivo. Studies in an in vivo zebrafish xenograft model further confirmed that administration of acRoots inhibits xenograft growth and metastasis of HGC-27 cells transplanted in vivo [34]. In addition, acRoots has been widely used in adjuvant therapy of tumors [19]. AcRoots-containing Chinese medicine formula "Weikang Keli" can significantly inhibit the proliferation of gastric cancer cells and prolong the survival period of nude mice by promoting autophagy [19]. Therefore, both in vitro and in vivo studies have proved that acRoots displayed good anticancer efficacy. However, in vivo efficacy and clinical effect of acRoots on BCa still need to be verified by further in vivo experimental models, which needs to be comprehensively considered according to the complex biological environment, and the physical and chemical properties, biological activity and stability of the acRoots.

In summary, we showed that acRoots inhibits cell proliferation, migration, invasion and EMT and induces apoptosis by attenuating the AKT/GSK-3 $\beta$ signaling pathway in $\mathrm{BCa}$ cells in vitro. The results demonstrated that acRoots may be a potential candidate for antitumor treatment.

\section{Acknowledgments}

Not applicable.

\section{Funding}

This study was supported by the Quzhou City Science And Technology Research Project (2018K23) and the Tianjin Natural Science Foundation (18JCQNJC13800).

\section{Availability of data and materials}

All data generated or analyzed during this study are included in this published article.

\section{Authors' contributions}

MNJ conceived and designed the experiments, CCG analyzed and interpreted the results of the experiments, and $\mathrm{ZJ}$ and XPW performed the experiments.

\section{Ethics approval and consent to participate}

Not applicable. 


\section{Patient consent for publication}

Not applicable.

\section{Informed consent}

Written informed consent was obtained from a legally authorized representative(s) for anonymized patient information to be published in this article.

\section{Conflict of interests}

The authors declare that they have no competing interests, and all authors should confirm its accuracy.

\section{References}

1. Torre LA, Bray F, Siegel RL, et al. Global cancer statistics, 2012. CA Cancer J Clin. 2015; 65(2): 87-108, doi: 10.3322/ caac.21262, indexed in Pubmed: 25651787.

2. Jemal A, Bray F, Center MM, et al. Global cancer statistics. CA Cancer J Clin. 2011; 61(2): 69-90, doi: 10.3322/caac.20107, indexed in Pubmed: 21296855.

3. Gonzalez-Angulo A, Morales-Vasquez F, Hortobagyi G. Overview of Resistance to Systemic Therapy in Patients with Breast Cancer. Breast Cancer Chemosensitivity. 2007: 1-22, doi: 10.1007/978-0-387-74039-3_1.

4. Cancer Genome Atlas Network. Comprehensive molecular portraits of human breast tumours. Nature. 2012; 490(7418): 61-70, doi: 10.1038/nature11412, indexed in Pubmed: 23000897.

5. Sutter SA, Slinker A, Balumuka DD, et al. Surgical Management of Breast Cancer in Africa: A Continent-Wide Review of Intervention Practices, Barriers to Care, and Adjuvant Therapy. J Glob Oncol. 2017; 3(2): 162-168, doi: 10.1200/ JGO.2016.003095, indexed in Pubmed: 28717754.

6. Caetano-Pinto P, Jansen J, Assaraf YG, et al. The importance of breast cancer resistance protein to the kidneys excretory function and chemotherapeutic resistance. Drug Resist Updat. 2017; 30: 15-27, doi: 10.1016/j.drup.2017.01.002, indexed in Pubmed: 28363332.

7. Tao Z, Shi A, Lu C, et al. Breast Cancer: Epidemiology and Etiology. Cell Biochem Biophys. 2015; 72(2): 333-338, doi: 10.1007/s12013-014-0459-6, indexed in Pubmed: 25543329.

8. Miller AB, Bulbrook RD. The epidemiology and etiology of breast cancer. N Engl J Med. 1980; 303(21): 1246-1248, doi: 10.1056/NEJM198011203032130, indexed in Pubmed: 7421960 .

9. Cragg GM, Newman DJ. Plants as a source of anti-cancer agents. J Ethnopharmacol. 2005; 100(1-2): 72-79, doi: 10.1016/j.jep.2005.05.011, indexed in Pubmed: 16009521.

10. Buyel JF. Plants as sources of natural and recombinant anti-cancer agents. Biotechnol Adv. 2018; 36(2): 506-520, doi: 10.1016/j.biotechadv.2018.02.002, indexed in Pubmed: 29408560.

11. Mitra S, Dash R. Natural Products for the Management and Prevention of Breast Cancer. Evid Based Complement Alternat Med. 2018; 2018: 8324696, doi: 10.1155/2018/8324696, indexed in Pubmed: 29681985.

12. Sun L, Li X, Li G, et al. Actinidia chinensis Planch. Improves the Indices of Antioxidant and Anti-Inflammation Status of Type 2 Diabetes Mellitus by Activating Keap1 and Nrf2 via the Upregulation of MicroRNA-424. Oxid Med Cell Longev. 2017; 2017: 1-14, doi: 10.1155/2017/7038789, indexed in Pubmed: 28642811.

13. Zhu WJ, Yu DH, Zhao M, et al. Antiangiogenic triterpenes isolated from Chinese herbal medicine Actinidia chinensis Planch. Anticancer Agents Med Chem. 2013; 13(2): 195-198, doi: 10.2174/1871520611313020002, indexed in Pubmed: 22934692.

14. Zhang L, Zhang W, Wang Q, et al. Purification, antioxidant and immunological activities of polysaccharides from Actinidia Chinensis roots. Int J Biol Macromol. 2015; 72: 975-983, doi: 10.1016/j.ijbiomac.2014.09.056, indexed in Pubmed: 25316423.

15. Hou J, Wang $\mathrm{L}, \mathrm{Wu} \mathrm{D}$. The root of Actinidia chinensis inhibits hepatocellular carcinomas cells through LAMB3. Cell Biol Toxicol. 2018; 34(4): 321-332, doi: 10.1007/s10565-0179416-7, indexed in Pubmed: 29127567.

16. Fang T, Hou J, He M, et al. Actinidia chinensis Planch root extract (acRoots) inhibits hepatocellular carcinoma progression by inhibiting EP3 expression. Cell Biol Toxicol. 2016; 32(6): 499-511, doi: 10.1007/s10565-016-9351-z, indexed in Pubmed: 27475644.

17. Fang T, Fang Y, Xu X, et al. Actinidia chinensis Planch root extract attenuates proliferation and metastasis of hepatocellular carcinoma by inhibiting epithelial-mesenchymal transition. J Ethnopharmacol. 2019; 231: 474-485, doi: 10.1016/j. jep.2018.11.014, indexed in Pubmed: 30415058.

18. He M, Hou J, Wang L, et al. Actinidia chinensis Planch root extract inhibits cholesterol metabolism in hepatocellular carcinoma through upregulation of PCSK9. Oncotarget. 2017; 8(26): 42136-42148, doi: 10.18632/oncotarget.15010, indexed in Pubmed: 28178673.

19. Huo J, Qin F, Cai X, et al. Chinese medicine formula "Weikang Keli" induces autophagic cell death on human gastric cancer cell line SGC-7901. Phytomedicine. 2013; 20(2): 159-165, doi: 10.1016/j.phymed.2012.10.001, indexed in Pubmed: 23141426.

20. Deng S, Hu B, An HM, et al. Teng-Long-Bu-Zhong-Tang, a Chinese herbal formula, enhances anticancer effects of 5-Fluorouracil in CT26 colon carcinoma. BMC Complement Altern Med. 2013; 13: 128, doi: 10.1186/1472-6882-13-128, indexed in Pubmed: 23758730.

21. Cheng QL, Li HL, Huang ZQ, et al. $2 \beta, 3 \beta$, 23-trihydroxyurs-12-ene-28-olic acid (TUA) isolated from Actinidia chinensis Radix inhibits NCI-H460 cell proliferation by decreasing NF- $\kappa$ B expression. Chem Biol Interact. 2015; 240: 1-11, doi: 10.1016/j.cbi.2015.06.038, indexed in Pubmed: 26134000.

22. Varankar SS, Bapat SA. Migratory Metrics of Wound Healing: A Quantification Approach for Scratch Assays. Front Oncol. 2018; 8: 633, doi: 10.3389/fonc.2018.00633, indexed in Pubmed: 30619767.

23. Sun L, Jin X, Xie L, et al. Swainsonine represses glioma cell proliferation, migration and invasion by reduction of miR92a expression. BMC Cancer. 2019; 19(1): 247, doi: 10.1186/ s12885-019-5425-7, indexed in Pubmed: 30890138.

24. Wang Z, Li TE, Chen Mo, et al. miR-106b-5p contributes to the lung metastasis of breast cancer via targeting CNN1 and regulating Rho/ROCK1 pathway. Aging (Albany NY). 2020; 12(2): 1867-1887, doi: 10.18632/aging.102719, indexed in Pubmed: 31986487.

25. Son YO, Wang L, Poyil P, et al. Cadmium induces carcinogenesis in BEAS-2B cells through ROS-dependent activation of PI3K/AKT/GSK-3 $\beta / \beta$-catenin signaling. Toxicol Appl Pharmacol. 2012; 264(2): 153-160, doi: 10.1016/j.taap.2012.07.028, indexed in Pubmed: 22884995. 
26. Zhou SL, Zhou ZJ, Hu ZQ, et al. CXCR2/CXCL5 axis contributes to epithelial-mesenchymal transition of HCC cells through activating PI3K/Akt/GSK-3 $\beta /$ Snail signaling. Cancer Lett. 2015; 358(2): 124-135, doi: 10.1016/j.canlet.2014.11.044, indexed in Pubmed: 25462858.

27. Chen Q, Yang D, Zong H, et al. Growth-induced stress enhances epithelial-mesenchymal transition induced by IL-6 in clear cell renal cell carcinoma via the Akt/GSK-3 $\beta / \beta$-catenin signaling pathway. Oncogenesis. 2017; 6(8): e375, doi: 10.1038/oncsis.2017.74, indexed in Pubmed: 28846080.

28. Liu J, Chen Y, Shuai S, et al. TRPM8 promotes aggressiveness of breast cancer cells by regulating EMT via activating AKT/ GSK-3 $\beta$ pathway. Tumour Biol. 2014; 35(9): 8969-8977, doi: 10.1007/s13277-014-2077-8, indexed in Pubmed: 24903376.

29. Ye X, Guo Yu, Zhang Qi, et al. $\beta$ Klotho suppresses tumor growth in hepatocellular carcinoma by regulating Akt/ GSK-3 / cyclin D1 signaling pathway. PLoS One. 2013; 8(1): e55615, doi: 10.1371/journal.pone.0055615, indexed in $\mathrm{Pu}-$ bmed: 23383245.

30. Lu J, Steeg PS, Price JE, et al. Breast cancer metastasis: challenges and opportunities. Cancer Res. 2009; 69(12): 4951-4953, doi: 10.1158/0008-5472.CAN-09-0099, indexed in Pubmed: 19470768.

31. Cohen I, Tagliaferri M, Tripathy D. Traditional Chinese medicine in the treatment of breast cancer. Semin Oncol. 2002; 29(6): 563-574, doi: 10.1053/sonc.2002.50005, indexed in Pubmed: 12516039.

32. Cui Y, Shu XO, Gao Y, et al. Use of complementary and alternative medicine by Chinese women with breast cancer. Breast Cancer Research and Treatment. 2004; 85(3): 263-270, doi: 10.1023/b:brea.0000025422.26148.8d.

33. Koff JL, Ramachandiran S, Bernal-Mizrachi L. A time to kill: targeting apoptosis in cancer. Int J Mol Sci. 2015; 16(2): 2942-2955, doi: 10.3390/ijms16022942, indexed in Pubmed: 25636036 .

34. Gao Z, Deng G, Li Y, et al. Actinidia chinensis Planch prevents proliferation and migration of gastric cancer associated with apoptosis, ferroptosis activation and mesenchymal phenotype suppression. Biomed Pharmacother. 2020; 126: 110092, doi: 10.1016/j.biopha.2020.110092, indexed in Pubmed: 32203890.

35. Ye X, Brabletz T, Kang Y, et al. Upholding a role for EMT in breast cancer metastasis. Nature. 2017; 547(7661): E1-E3, doi: 10.1038/nature22816, indexed in Pubmed: 28682326.

36. Gunasinghe NP, Wells A, Thompson EW, et al. Mesenchymal-epithelial transition (MET) as a mechanism for metastatic colonisation in breast cancer. Cancer Metastasis Rev. 2012; 31(3-4): 469-478, doi: 10.1007/s10555-012-9377-5, indexed in Pubmed: 22729277.

37. Wang Y, Shi J, Chai K, et al. The Role of Snail in EMT and Tumorigenesis. Curr Cancer Drug Targets. 2013; 13(9): 963-972, doi: 10.2174/15680096113136660102, indexed in Pubmed: 24168186.

38. Berx G, Van Roy F. The E-cadherin/catenin complex: an important gatekeeper in breast cancer tumorigenesis and malignant progression. Breast Cancer Res. 2001; 3(5): 289-293, doi: 10.1186/bcr309, indexed in Pubmed: 11597316.

39. Mrozik KM, Blaschuk OW, Cheong CM, et al. N-cadherin in cancer metastasis, its emerging role in haematological malignancies and potential as a therapeutic target in cancer. BMC Cancer. 2018; 18(1): 939, doi: 10.1186/s12885-018-4845-0, indexed in Pubmed: 30285678.

40. Lv J, Wang L, Shen H, et al. Regulatory roles of OASL in lung cancer cell sensitivity to Actinidia chinensis Planch root extract (acRoots). Cell Biol Toxicol. 2018; 34(3): 207-218, doi: 10.1007/s10565-018-9422-4, indexed in Pubmed: 29508110.

41. Wang Q, Xu Y, Gao Y, et al. Actinidia chinensis planch polysaccharide protects against hypoxiainduced apoptosis of cardiomyocytes in vitro. Molecular Medicine Reports. 2018, doi: 10.3892/mmr.2018.8953, indexed in Pubmed: 29750308.

42. Cohen P, Frame S. The renaissance of GSK3. Nat Rev Mol Cell Biol. 2001; 2(10): 769-776, doi: 10.1038/35096075, indexed in Pubmed: 11584304.

43. Sharma M, Chuang WW, Sun Z. Phosphatidylinositol 3-kinase/Akt stimulates androgen pathway through GSK3beta inhibition and nuclear beta-catenin accumulation. J Biol Chem. 2002; 277(34): 30935-30941, doi: 10.1074/jbc.M201919200, indexed in Pubmed: 12063252.

44. MacDonald BT, Tamai K, He Xi. Wnt/beta-catenin signaling: components, mechanisms, and diseases. Dev Cell. 2009; 17(1): 9-26, doi: 10.1016/j.devcel.2009.06.016, indexed in Pubmed: 19619488.

45. Li D, Beisswenger C, Herr C, et al. Myeloid cell RelA/p65 promotes lung cancer proliferation through $\mathrm{Wnt} / \beta$-catenin signaling in murine and human tumor cells. Oncogene. 2014; 33(10): 1239-1248, doi: 10.1038/onc.2013.75, indexed in Pubmed: 23563178 .

46. Qu T, Zhao Y, Chen Y, et al. Down-regulated MAC30 expression inhibits breast cancer cell invasion and EMT by suppressing $\mathrm{Wnt} / \beta$-catenin and PI3K/Akt signaling pathways. Int J Clin Exp Pathol. 2019 ; 12(5): 1888-1896, indexed in Pubmed: 31934012.

Submitted: 4 March, 2021

Accepted after reviews: 6 January, 2021 Available as AoP: 30 January, 2021 